\title{
Gravity waves from inflating brane or Mirrors moving in $\operatorname{adS}_{5}$
}

\author{
D.S. Gorbunov 周, V.A. Rubakov 币, S.M. Sibiryakov 尚 \\ Institute for Nuclear Research of the Russian Academy of Sciences, Moscow 117312, Russia
}

\begin{abstract}
We study tensor perturbations in a model with inflating Randall-Sundrum-type brane embedded in five-dimensional anti-de Sitter $\left(\mathrm{adS}_{5}\right)$ bulk. In this model, a natural in-vacuum of gravitons is the vacuum defined in static $\mathrm{adS}_{5}$ frame. We show that this vacuum is, in fact, the same as the in-vacuum defined in the frame with de Sitter $\left(\mathrm{dS}_{4}\right)$ slicing, in which the brane is at rest. Thus, during inflation, gravitons on and off the brane remain in their vacuum state. We then study graviton creation by the brane on which inflation terminates at some moment of time. We mostly consider gravitons whose wavelengths at the end of inflation exceed both the horizon size and the $\operatorname{adS}_{5}$ radius. Creation of these gravitons is dominated by (zero mode)-(zero mode) Bogoliubov coefficients and, apart from an overall factor, the spectrum of produced gravitons is the same as in four-dimensional theory. "Kaluza-Klein" gravitons are also produced, but this effect is subdominant. Graviton spectra at somewhat higher momenta are also presented for completeness.
\end{abstract}

\footnotetext{
*E-mail: gorby@ms2.inr.ac.ru

${ }^{\dagger}$ E-mail: rubakov@ms2.inr.ac.ru

${ }^{\ddagger}$ E-mail: sibir@ms2.inr.ac.ru
} 


\section{Introduction and summary}

A special property of brane-world models with infinite extra dimension(s), namely, the Randall-Sundrum (RS) model [1] and its generalizations (for a review see, e.g., Ref. [2]) is the existence of soft bulk gravitons with continuum spectrum of four-dimensional masses. This property is of interest in the cosmological context, as in principle it may affect the spectrum of long-wavelength perturbations created at inflation, and therefore the large scale structure and CMB anisotropy. In inflationary models, perturbations which are of long-wavelengths today, are created from zero-point fluctuations of high initial momenta, the corresponding "Kaluza-Klein" (KK) zero-point fluctuations are generically not suppressed near the brane at early times, and thus may not be negligible. Also, if soft KK gravitons were abundantly produced at inflation and survived until recent epoch, they would leave footprints in CMB anisotropy.

The simplest inflationary brane-world model is that of inflating RS brane embedded in the five-dimensional anti-de Sitter $\left(\mathrm{adS}_{5}\right)$ bulk. Naturally, considerable effort has been put into the study of the generation of perturbations in this model [3, 4, 5], in particular, the tensor perturbations «, [5. The latter analyses utilized either brane-based approach [5] or adS/CFT correspondence [4]. However, the most straightforward approach to the study of the generation of tensor perturbations, which we adopt in this paper, is to solve the five-dimensional linearized Einstein equations with appropriate boundary conditions on the brane, and calculate the Bogoliubov coefficients. Such a calculation is similar to the study of particle creation by moving mirrors: in static ad $\mathrm{S}_{5}$ coordinates, the three-brane moves [6] and acts as a mirror (with Neumann boundary conditions) for five-dimensional gravitons. The calculation of this sort enables one, at least in principle, to evaluate the spectrum of created gravitons (not only in the zero-mode final state, as in Ref. [5], but also in continuum states) and also to find the relevant correlators (not only at the de Sitter stage of the brane evolution, as in Ref. [由], but also after inflation).

In this paper we first consider an inflating brane with de Sitter $\left(\mathrm{dS}_{4}\right)$ intrinsic geometry, 
which is a boundary of a part of $\operatorname{adS}_{5}$. We address the following issue. The natural graviton vacuum in $\mathrm{adS}_{5}$, cut by the brane, is the state which does not contain five-dimensional gravitons moving towards the brane (we refer to this state, somewhat loosely, as ad $\mathrm{S}_{5^{-}}$ vacuum). This definition needs qualification, as it involves the choice of a coordinate frame in $\mathrm{adS}_{5}$. The appropriate frame is static, so the brane moves in this frame. From the point of view of an observer residing on the brane, another coordinate frame in $\operatorname{adS}_{5}$ is appropriate, in which the brane is at rest. The latter frame, which corresponds to $\mathrm{dS}_{4}$ slicing of $\operatorname{adS}_{5}$, defines its own graviton vacuum, which may or may not coincide with the $\mathrm{adS}_{5}$-vacuum. In Section 2 we find that, in fact, the vacuum defined with respect to $\mathrm{dS}_{4}$ slicing is the same as $\mathrm{adS}_{5}$-vacuum. This means that the fact that the $i n$-vacuum is defined in static ad $\mathrm{S}_{5}$ frame does not add any physics above and beyond that inherent in $\mathrm{dS}_{4}$ slicing; gravitons on and off the brane remain in their vacuum state. This situation is in clear contrast to the case of a mirror accelerating in Minkowski space-time. Our result in a sense contradicts expectations expressed in Ref. [7], but we think it is natural. Indeed, consider a brane on which matter in its ground state has energy density in excess to the fine-tuned RS value. Then there is no reason to suspect that the system would excite itself, i.e., it is natural that quantum fields (including gravitons) remain in their ground state, and the expansion of the brane is precisely de Sitter円.

Technically, our calculation involves the evaluation of the Bogoliubov coefficients relating two sets of graviton wave functions: one set consists of waves moving towards the brane, with frequency decomposition defined in the static adS $\mathrm{S}_{5}$ frame, while another set [8, 5, 9] has fixed frequencies in the frame corresponding to $\mathrm{dS}_{4}$ slicing. We find that the non-trivial Bogoliubov coefficients vanish, i.e., the wave functions which are negative frequency in one frame, do not have positive-frequency components in another frame.

In Section 3 we proceed to calculate the graviton creation by the brane on which inflation terminates at some moment of time. We are interested mostly in gravitons whose

\footnotetext{
${ }^{1}$ In the case of accelerated mirror in Minkowski space-time, there is external force that accelerates the mirror. Such external force is absent in our case.
} 
wavelengths at the end of inflation are larger than both the $\mathrm{dS}_{4}$ horizon size $H^{-1}$ and adS radius $k^{-1}$. We argue in Appendix 1 that for all realistic adS $S_{5}$ radii, only this part of the spectrum is relevant for the generation of CMB anisotropy at interesting angular scales.

We find no surprizes. The creation of large wavelength gravitons is dominated by (zero mode)-(zero mode) Bogoliubov coefficients and, apart from the overall enhancement factor [5], the spectrum of produced gravitons is the same as in four-dimensional theories. The continuum KK gravitons are also produced, but their number is suppressed. As anticipated in Ref. [5], KK part of initial zero-point fluctuations gives negligible contributions into production of gravitons in both zero and continuum modes. Hence, the spectrum of large wavelength gravitons, created due to inflation on the brane, is indistinguishable from the spectrum predicted by four-dimensional theories.

If the Hubble parameter on the inflating brane, $H$, is much larger than the inverse $\operatorname{adS}_{5}$ radius $k$, there is a range of graviton spatial momenta at the end of inflation, $p_{\text {phys }}$, in which the gravitons are superhorizon at this moment of time, but their wavelengths are

smaller than the $\operatorname{adS}_{5}$ radius, $H \gg p_{\text {phys }} \gg k$. For completeness, we consider creation of these gravitons in Appendix 4. We find that the spectrum of created zero-mode gravitons gets damped at these momenta. One peculiar feature of this case is that the creation of KK gravitons in the final state dominates over the production of zero-mode gravitons, in contrast to the case of soft gravitons studied in the main text. Still, the energy density emitted into the bulk in the part of the spectrum $H \gg p_{\text {phys }} \gg k$ is a small fraction of the energy density on the brane. The emission of gravitons into the bulk is a small effect at least for gravitons which are superhorizon by the end of inflation.

\section{$2 \quad \mathrm{dS}_{4}$ mirror moving in $\operatorname{adS}_{5}$}




\section{$2.1 \quad$ in-vacuum in $\operatorname{adS}_{5}$}

The $\mathrm{adS}_{5}$ metric in static coordinates is

$$
d s^{2}=\frac{1}{(k z)^{2}}\left(d t^{2}-d \mathbf{x}^{2}-d z^{2}\right)
$$

A spatially flat three-brane moving in these coordinates is described by a world surface

$$
t=t(\eta), \quad z=z(\eta)
$$

where $\eta$ is the intrinsic time coordinate on the brane.

Throughout this paper we consider tensor perturbations, i.e., write the perturbed metric as follows,

$$
d s^{2}=\frac{1}{(k z)^{2}}\left[d t^{2}-\left(\delta_{i j}+h_{i j}\right) d x^{i} d x^{j}-d z^{2}\right]
$$

where $h_{i j}$ are transverse and traceless. The perturbations are decomposed in spatial Fourier modes

$$
h_{i j}(t, \mathbf{x}, z)=\mathrm{e}^{i \mathbf{p x}} e_{i j} \Phi(t, z ; p)
$$

where $e_{i j}$ are constant transverse-traceless polarization tensors, and $\Phi$ obeys the KleinGordon equation for minimal massless scalar field in $\operatorname{adS}_{5}$ [3], 5],

$$
\left(-\frac{\partial^{2}}{\partial z^{2}}+\frac{3}{z} \frac{\partial}{\partial z}+\frac{\partial^{2}}{\partial t^{2}}+p^{2}\right) \Phi=0
$$

With $Z_{2}$ orbifold symmetry imposed across the brane, the boundary condition for $\Phi$ is Neumann on the brane,

$$
\partial_{n} \Phi=0
$$

where $\partial_{n}$ denotes the derivative normal to the brane.

The in-vacuum in $\mathrm{adS}_{5}$ is defined by choosing the basis of incoming waves (falling towards the brane) which have fixed frequencies in the static frame. The solutions to eq. (5)), which are incoming waves at large $z$ and have fixed frequencies, are (up to normalization,

\footnotetext{
${ }^{2}$ These solutions do not obey the Neumann boundary conditions (6) on the brane. However, as long as one considers asymptotic past, one can use wave packets composed of these functions, and disregard this subtlety.
} 
which is unimportant for our purposes)

$$
\phi_{i n-a d S, m}^{(+)}=\mathrm{e}^{i \omega t} z^{2} H_{2}^{(1)}(m z)
$$

and

$$
\phi_{i n-a d S, m}^{(-)}=\left(\phi_{i n-a d S, m}^{(+)}\right)^{*}=\mathrm{e}^{-i \omega t} z^{2} H_{2}^{(2)}(m z)
$$

where $H_{2}^{(1,2)}$ are the Hankel functions, the continuous parameter $m$ is positive, $m>0$, and

$$
\omega=\sqrt{m^{2}+p^{2}}
$$

These solutions behave at large $z$ as follows

$$
\phi_{\text {in-adS, } m}^{( \pm)}=-\sqrt{\frac{2}{\pi m}} \cdot z^{3 / 2} \mathrm{e}^{ \pm i(\omega t+m z-\pi / 4)}
$$

As a side remark, we note that the normalization condition is, generally speaking,

$$
\int d z \sqrt{g} g^{00} i\left(\phi_{m^{\prime}}^{*} \partial_{t} \phi_{m}-\partial_{t} \phi_{m^{\prime}}^{*} \phi_{m}\right)=\delta_{m^{\prime}, m}
$$

The factor $z^{3 / 2}$ in eq. (10) is precisely what is needed to normalize these solutions to deltafunction $\delta\left(m^{\prime}-m\right)$ with the weight $\sqrt{g} g^{00} \propto z^{-3}$.

\section{$2.2 \quad \mathrm{dS}_{4}$ slicing}

The frame in which $\mathrm{dS}_{4}$ brane is static is obtained by introducing coordinates $\eta<0$ and $\xi$, related to $t$ and $z$ as follows

$$
\begin{aligned}
& t=\eta \cosh \xi \\
& z=-\eta \sinh \xi
\end{aligned}
$$

In these coordinates, the $\operatorname{adS}_{5}$ metric is

$$
d s^{2}=\frac{1}{(k \sinh \xi)^{2}}\left[\frac{1}{\eta^{2}}\left(d \eta^{2}-d \mathbf{x}^{2}\right)-d \xi^{2}\right]
$$


The $\mathrm{dS}_{4}$ brane is at constant $\xi$,

$$
\xi=\xi_{B}
$$

i.e. in static coordinates it moves along the straight line

$$
\begin{aligned}
t & =\eta \cosh \xi_{B} \\
z & =-\eta \sinh \xi_{B}
\end{aligned}
$$

It is clear from eqs. (12) and (13) that the induced metric on the brane is indeed de Sitter, the Hubble parameter on the brane is

$$
H=k \sinh \xi_{B}
$$

and the coordinate $\eta$ is the conformal time in $\mathrm{dS}_{4}$.

In the new coordinates, the Klein-Gordon equation is

$$
\left[\left(\frac{\partial^{2}}{\partial \eta^{2}}-\frac{2}{\eta} \frac{\partial}{\partial \eta}\right)-\frac{1}{\eta^{2}}\left(\frac{\partial^{2}}{\partial \xi^{2}}-\frac{3 \cosh \xi}{\sinh \xi} \frac{\partial}{\partial \xi}\right)+p^{2}\right] \Phi=0
$$

Since the brane is static, the boundary condition (6) takes a simple form,

$$
\left.\partial_{\xi} \Phi\right|_{\xi=\xi_{B}}=0
$$

One solution to these equations is the bound state (zero mode), $\phi_{0}(\eta)$, which depends only on $\eta$. The zero mode which in infinite past has negative frequency with respect to time $\eta$ is

$$
\phi_{0}^{(-)}=k^{\frac{3}{2}} \sinh \xi_{B} \frac{C_{1}}{\sqrt{p}} \mathrm{e}^{-i p \eta}\left(\eta-\frac{i}{p}\right)
$$

where the normalization factor $C_{1}$ is determined by eq. (11) written in $(\eta, \xi)$ frame,

$$
\int \frac{d \xi}{(k \sinh \xi)^{3} \eta^{2}} i\left(\phi_{\kappa^{\prime}}^{*} \partial_{\eta} \phi_{\kappa}-\partial_{\eta} \phi_{\kappa^{\prime}}^{*} \phi_{\kappa}\right)=\delta_{\kappa^{\prime}, \kappa}
$$

Here $\kappa$ labels the solutions to eqs. (16), (17); for the moment $\kappa=\kappa^{\prime}=0$. One finds from eq. (19)

$$
C_{1}^{2} \cdot 2\left(\sinh \xi_{B}\right)^{2} \int_{\xi_{B}}^{\infty} \frac{d \xi}{\sinh ^{3} \xi}=1
$$


The quantity $C_{1}$ is precisely the same as that introduced in Ref. [5]. In the case of slow inflation, $H \ll k$ (and hence $\xi_{B} \ll 1$ ), one has $C_{1}=1$, while at $H \gg k$ one finds $C_{1}=$ $\sqrt{3 H / 2 k}$.

Let us now consider non-zero modes of eq.(16). The variables decouple, so the modes have the form

$$
\phi_{\kappa}(\eta, \xi)=\psi_{\kappa}(\eta) \cdot \chi_{\kappa}(\xi)
$$

where $\psi_{\kappa}$ obeys

$$
\left(\frac{\partial^{2}}{\partial \eta^{2}}-\frac{2}{\eta} \frac{\partial}{\partial \eta}+\frac{\kappa^{2}+\frac{9}{4}}{\eta^{2}}+p^{2}\right) \psi_{\kappa}=0
$$

whereas the spatial part of the wave function obeys the boundary condition (17) and equation

$$
\left(\frac{\partial^{2}}{\partial \xi^{2}}-\frac{3 \cosh \xi}{\sinh \xi} \frac{\partial}{\partial \xi}+\kappa^{2}+\frac{9}{4}\right) \chi_{\kappa}=0
$$

Equation (22) may be reduced to the Bessel equation. Its solution which is negativefrequency at large negative $\eta$ is

$$
\psi_{\kappa}^{(-)}(\eta)=\frac{\sqrt{\pi}}{2} k^{\frac{3}{2}} \mathrm{e}^{\frac{\pi \kappa}{2}}|\eta|^{\frac{3}{2}} H_{i \kappa}^{(2)}(p \eta)
$$

At large negative $\eta$ it behaves as follows

$$
\psi_{\kappa}^{(-)}=-\frac{1}{\sqrt{2 p}} k^{\frac{3}{2}} \eta \mathrm{e}^{-i p \eta-i \pi / 4}
$$

The normalization factor is chosen in such a way that

$$
\frac{1}{k^{3} \eta^{2}} i\left(\psi_{\kappa}^{(+)} \partial_{\eta} \psi_{\kappa}^{(-)}-\text {c.c. }\right)=1
$$

(where $\left.\psi_{\kappa}^{(+)}=\left(\psi_{\kappa}^{(-)}\right)^{*}\right)$. The factor $k^{-3} \eta^{-2}$ here comes from the measure in (19).

The spatial wave functions $\chi_{\kappa}$ are considered in detail in Appendix 2. Their form is not important in this section. It is worth pointing out, however, that there are no bound states except for the zero mode, and that the parameter $\kappa$ is continuous and starts from zero. 


\subsection{Vacuum in $\mathrm{dS}_{4}$ slicing coincides with ad $\mathrm{S}_{5}$-vacuum}

Let us see that the wave functions $\phi_{\kappa}^{(-)}$, which are negative frequency with respect to time $\eta$, are also negative frequency with respect to time $t$. This will mean that the graviton vacua defined in the two frames (11) and (12), respectively, are in fact the same

We are going to calculate the Bogoliubov coefficients between the sets $\phi_{\kappa}^{(-)}$and $\phi_{\text {in-adS,m }}^{(+)} \equiv\left(\phi_{\text {in-adS,m }}^{(-)}\right)^{*}$. These are given by scalar products similar to the left hand sides of eqs. (11) or (19), but now involving the functions $\phi_{\kappa}^{(-)}$and $\phi_{i n-a d S, m}^{(+)}$. We prefer to integrate over the hypersurface of fixed $\eta$, and then take the limit $\eta \rightarrow-\infty$. Hence the Bogoliubov coefficients of interest are proportional to

$$
v_{\kappa, m}^{d S-a d S} \propto \int_{\eta \rightarrow-\infty} \frac{d \xi}{(\sinh \xi)^{3} \eta^{2}}\left(\phi_{\kappa}^{(-)} \partial_{\eta}\left[\phi_{i n-a d S, m}^{(+)}\right]^{*}-\left[\phi_{i n-a d S, m}^{(+)}\right]^{*} \partial_{\eta} \phi_{\kappa}^{(-)}\right)
$$

where the $\operatorname{adS}_{5}$ wave functions are expressed through coordinates $\eta$ and $\xi$,

$$
\left[\phi_{i n-a d S, m}^{(+)}\right]^{*} \equiv \phi_{i n-a d S, m}^{(-)}=\mathrm{e}^{-i \omega \eta \cosh \xi}(\eta \sinh \xi)^{2} H_{2}^{(2)}(-m \eta \sinh \xi)
$$

Let us first note that at large negative $\eta$, both the zero mode $\phi_{0}^{(-)}$and non-zero modes $\phi_{\kappa}^{(-)}$, corresponding to $\mathrm{dS}_{4}$ slicing, behave in a similar way,

$$
\phi_{\kappa}^{(-)}=\eta \mathrm{e}^{-i p \eta} \chi_{\kappa}(\xi)
$$

At large negative $\eta$, the integrand in eq. (27) rapidly oscillates, and we may use the saddlepoint approximation. The saddle point $\xi_{s}$ is finite, so at large $|\eta|$ we use the asymptotic form of the Hankel function $H_{2}^{(2)}(-m \eta \sinh \xi)$, see also (10). In this way we obtain

$$
\begin{aligned}
v_{\kappa, m}^{d S-a d S} \propto \sqrt{|\eta|} \int_{\eta \rightarrow-\infty} & \frac{d \xi}{\sinh ^{3 / 2} \xi} \chi_{\kappa}(\xi) \mathrm{e}^{-i \omega \eta \cosh \xi+i m \eta \sinh \xi} \mathrm{e}^{-i p \eta} \times \\
& {\left[-\omega \cosh \xi+m \sinh \xi+p+O\left(\eta^{-1}\right)\right] }
\end{aligned}
$$

\footnotetext{
${ }^{3}$ There is a subtlety here. The $\mathrm{dS}_{4}$ slicing (12) and correspondingly the wave functions $\phi_{\kappa}$ are defined only in the past light cone, $z<-t$. As long as physics at or near the brane is considered, this subtlety is irrelevant.
} 
The saddle point is at

$$
\tanh \xi_{s}=\frac{m}{\omega}
$$

that is

$$
\sinh \xi_{s}=\frac{m}{p}, \quad \cosh \xi_{s}=\frac{\omega}{p}
$$

At this point, however, the pre-factor vanishes, up to possible corrections decaying as $\eta^{-1}$. Thus

$$
v_{\kappa, m}^{d S-a d S}=0
$$

as promised.

A remark is in order. Should we calculate $u$-coefficients, relating components $\phi_{k}^{( \pm)}$and $\phi_{i n-a d S, m}^{( \pm)}$of the same sign of frequencies, the pre-factor would not vanish at the saddle point, and we would obtain the result which is finite as $\eta \rightarrow-\infty$ (the overall factor $\sqrt{|\eta|}$ in (30) would cancel $|\eta|^{-1 / 2}$ which would come from the Gaussian integration near the saddle point). This may be viewed as a cross check of our calculation.

\section{Gravity waves from inflation on the brane}

\subsection{A model for the background}

The main purpose of this paper is to calculate the spectrum of gravitons created by the brane which inflates for long period of time, and then enters the Friedmann regime. As long as one is interested in gravitons which are superhorizon by the end of inflation, it suffices to use the following simplified model for the background. We consider the brane which inflates until some moment of time $\eta_{0}<0$, and then does not evolve at all. In a similar model in four dimensions, the calculation of graviton creation is very simple, and is presented in Appendix 3. Of course, its result coincides with the result of realistic calculations which take into account evolution of the Universe after inflation. 
In static coordinates the trajectory of the brane is the straight line (14) for $\eta<\eta_{0}$, and then another straight line parallel to the $t$-axis,

$$
z=z_{B}=\left|\eta_{0}\right| \sinh \xi_{B}
$$

as shown in Figure 1. The brane after inflation is Minkowskian, with the scale factor on the

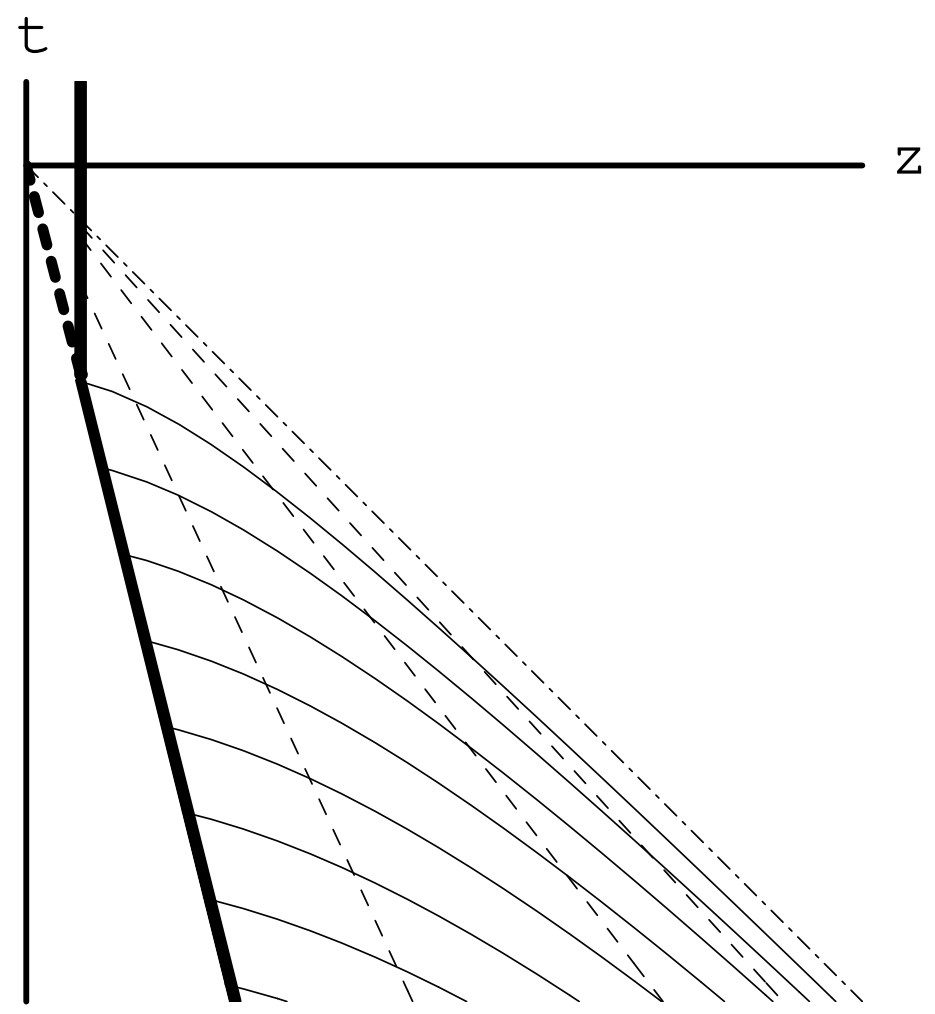

Figure 1: Brane motion in static coordinates: thick solid line represents the world surface of the brane, dash-dotted line denotes the past light cone; thin solid (dashed) lines correspond to surfaces of fixed $\eta(\xi)$.

brane being

$$
a\left(z_{B}\right)=\frac{1}{k\left|\eta_{0}\right| \sinh \xi_{B}}
$$

Of course, one could rescale $\eta$ in such a way that the scale factor on Minkowski brane is equal to 1 , but it is convenient to keep $\eta_{0}$ arbitrary, and make sure, as a cross check, that 
the results depend on physical quantities. Namely, the physical momentum at the end of inflation (and on Minkowski brane) is related to the coordinate momentum $p$ as follows,

$$
p_{\text {phys }}=\frac{p}{a\left(z_{B}\right)}=p k\left|\eta_{0}\right| \sinh \xi_{B}
$$

Comparing this expression with eq. (15), we find that the modes which are superhorizon at the end of inflation, obey

$$
\frac{p_{\text {phys }}}{H} \equiv p\left|\eta_{0}\right| \ll 1
$$

We will be interested mostly in modes whose physical momentum at the end of inflation is small compared to the inverse $\operatorname{adS}_{5}$ radius, see Appendix 1. In terms of the coordinate momentum, this means

$$
\frac{p_{\text {phys }}}{k} \equiv p\left|\eta_{0}\right| \sinh \xi_{B} \ll 1
$$

In view of these relations, it will be convenient to consider $\left|\eta_{0}\right|$ as a small parameter of the problem.

We note in passing that at $H \gg k$, the spatial momenta of superhorizon modes may be such that the relation (38) is violated. Though the corresponding range of momenta,

$H \gg p_{\text {phys }} \gg k$, is not particularly interesting in the cosmological context, we consider this range in Appendix 4 for completeness.

\subsection{In-out Bogoliubov coefficients: generalities}

We are going to calculate the Bogoliubov coefficients which relate the graviton wave functions corresponding to in- and out-vacua. The wave functions of the $i n$-vacuum have been already described. In our simplified model with Minkowski brane at late times, the wave functions of out-vacuum are the standard RS wave functions (modulo factors accounting for the fact that the brane is placed at $z=z_{B}$ ). Namely, the normalized zero mode is

$$
\varphi_{0}^{( \pm)}=z_{B} \frac{k^{\frac{3}{2}}}{\sqrt{p}} \mathrm{e}^{ \pm i p t}
$$


Non-zero modes are

$$
\varphi_{m}^{( \pm)}(z, t)=\frac{k^{\frac{3}{2}}}{\sqrt{2 \omega}} \mathrm{e}^{ \pm i \omega t} \varphi_{m}(z)
$$

where

$$
\varphi_{m}(z)=z^{2} \sqrt{\frac{m}{2}} \frac{N_{1}\left(m z_{B}\right) J_{2}(m z)-J_{1}\left(m z_{B}\right) N_{2}(m z)}{\sqrt{\left[N_{1}\left(m z_{B}\right)\right]^{2}+\left[J_{1}\left(m z_{B}\right)\right]^{2}}}
$$

These spatial functions are normalized as follows,

$$
\int_{z_{B}}^{\infty} \frac{d z}{z^{3}} \varphi_{m} \varphi_{m^{\prime}}=\delta\left(m-m^{\prime}\right)
$$

the factor $k^{3 / 2}$ in the expressions (39) and (40) is due to the measure in (11).

Soft KK gravitons have low spatial momentum and frequency (and hence mass $m$ ). So, we assume in the main text that, together with relations (37) and (38), their counterparts

$$
m\left|\eta_{0}\right| \ll 1
$$

and

$$
m\left|\eta_{0}\right| \sinh \xi_{B} \ll 1
$$

are satisfied.

One way to calculate the Bogoliubov coefficients between in- and out-vacua is to specify the solution (of fixed frequency) at infinite future, evolve it backwards in time and find its decomposition in terms of the wave functions corresponding to $i n$-vacuum. Since negative frequency functions in the frame with $\mathrm{dS}_{4}$ slicing are at the same time negative frequency functions of $\operatorname{adS}_{5}$ vacuum, we are free to use either of these two sets as defining in-vacuum (provided we consider the interior of past light cone)

Thus, the solution of interest coincides in the future (flat brane) region with one of the RS wave functions $\varphi$. For the solution everywhere in space-time bounded by the brane we write

$$
\Phi=\varphi+\Delta \Phi
$$

Clearly, the first term here does not contribute to the Bogoliubov $v$-coefficients, relating inand out-functions of different frequencies (the RS wave functions have fixed frequencies in 
static adS $\mathrm{S}_{5}$ coordinates), so the quantity of interest is $\Delta \Phi$. Using Green's formula, we write

$$
\Delta \Phi(\eta, \xi)=-\int D_{a d v}\left(\eta, \xi ; \eta^{\prime}, \xi_{B}\right)\left[\partial_{\xi^{\prime}} \varphi\left(\eta^{\prime}, \xi^{\prime}\right)\right]_{\xi^{\prime}=\xi_{B}} d \eta^{\prime}
$$

The integration here is performed along the de Sitter brane, i.e. $\eta^{\prime}<\eta_{0}$. The extra term $\Delta \Phi$ concentrates in the past light cone, so we may use the set of the wave functions obtained with $\mathrm{dS}_{4}$ slicing, eqs. (18) and (21), as the set defining the in-vacuum.

The advanced propagator obeys

$$
\left[\left(\eta^{2} \frac{\partial^{2}}{\partial \eta^{2}}-2 \eta \frac{\partial}{\partial \eta}+p^{2} \eta^{2}\right)-\mathcal{O}_{\xi}\right] D_{a d v}\left(\eta, \xi ; \eta^{\prime}, \xi^{\prime}\right)=\delta\left(\xi-\xi^{\prime}\right) \delta\left(\eta-\eta^{\prime}\right)
$$

where

$$
\mathcal{O}_{\xi}=\frac{\partial^{2}}{\partial \xi^{2}}-3 \frac{\cosh \xi}{\sinh \xi} \frac{\partial}{\partial \xi}
$$

The form of eq.(47) is chosen in such a way that the Geen's formula holds without any additional measure (in particular, the operator $\partial_{\xi}^{2}$ enters eq.47) without any pre-factor).

Taking into account that the operator $\mathcal{O}_{\xi}$ is Hermitean (on wave functions satisfying the Neumann condition at $\xi=\xi_{B}$ ) with weight $\sinh ^{-3} \xi$ we obtain

$$
D_{a d v}\left(\eta, \xi ; \eta^{\prime}, \xi^{\prime}\right)=\sum_{\kappa} \chi_{\kappa}(\xi) \chi_{\kappa}\left(\xi^{\prime}\right) \frac{1}{\sinh ^{3} \xi^{\prime}} D_{\kappa}\left(\eta, \eta^{\prime}\right)
$$

where

$$
D_{\kappa}\left(\eta, \eta^{\prime}\right)=i \frac{1}{k^{3}} \theta\left(\eta^{\prime}-\eta\right)\left[\psi_{\kappa}^{(+)}(\eta) \psi_{\kappa}^{(-)}\left(\eta^{\prime}\right)-\psi_{\kappa}^{(-)}(\eta) \psi_{\kappa}^{(+)}\left(\eta^{\prime}\right)\right] \frac{1}{\eta^{\prime 4}}
$$

Here $\chi_{\kappa}$ and $\psi_{\kappa}^{( \pm)}$are space and time components of the graviton wave functions (including the zero mode) in the frame with $\mathrm{dS}_{4}$ slicing, see eqs. (18) and (21).

Collecting all factors, we write

$$
\begin{aligned}
& \Delta \Phi(\eta, \xi)=-\frac{i}{k^{3}} \sum_{\kappa} \int \frac{d \eta^{\prime}}{\sinh ^{3} \xi_{B} \eta^{\prime 4}} \theta\left(\eta^{\prime}-\eta\right) \times \\
& {\left[\phi_{\kappa}^{(+)}(\eta, \xi) \phi_{\kappa}^{(-)}\left(\eta^{\prime}, \xi_{B}\right)-\phi_{\kappa}^{(-)}(\eta, \xi) \phi_{\kappa}^{(+)}\left(\eta^{\prime}, \xi_{B}\right)\right] \times } \\
& {\left[\partial_{\xi^{\prime}} \varphi\left(\eta^{\prime}, \xi^{\prime}\right)\right]_{\xi^{\prime}=\xi_{B}} }
\end{aligned}
$$


where $\phi_{\kappa}^{( \pm)}(\eta, \xi)$ are the total wave functions in the frame with $\mathrm{dS}_{4}$ slicing. These are given by expressions (18) and (21).

The advantage of this formula is that the Bogoliubov coefficients follow immediately. For

example, take $\varphi=\varphi_{m}^{(-)}$. Then the $v$-coefficient, that determines the creation of gravitons in this mode from the zero-point fluctuations in the mode $\phi_{\kappa}$, is the coefficient in front of $\phi_{\kappa}^{(+)}(\eta, \xi)$ in eq. (51). Namely, we take the limit $\eta \rightarrow-\infty$ and obtain

$$
v_{m, \kappa}=-\frac{i}{k^{3}} \int \frac{d \eta^{\prime}}{\sinh ^{3} \xi_{B} \eta^{\prime 4}} \phi_{\kappa}^{(-)}\left(\eta^{\prime}, \xi_{B}\right)\left[\partial_{\xi^{\prime}} \varphi_{m}^{(-)}\left(\eta^{\prime}, \xi^{\prime}\right)\right]_{\xi^{\prime}=\xi_{B}}
$$

or explicitly (and omitting unnecessary primes)

$$
v_{m, \kappa}=-\frac{i}{k^{3}} \frac{\chi_{\kappa}\left(\xi_{B}\right)}{\sinh ^{3} \xi_{B}} \int_{-\infty}^{\eta_{0}} \frac{d \eta}{\eta^{4}} \psi_{\kappa}^{(-)}(\eta)\left[\partial_{\xi} \varphi_{m}^{(-)}(\eta, \xi)\right]_{\xi=\xi_{B}}
$$

Similarly, the $u$-coefficients relating the wave functions with the same signs of frequencies, are obtained as follows,

$$
u_{m, \kappa}=-\frac{i}{k^{3}} \frac{\chi_{\kappa}\left(\xi_{B}\right)}{\sinh ^{3} \xi_{B}} \int_{-\infty}^{\eta_{0}} \frac{d \eta}{\eta^{4}} \psi_{\kappa}^{(-)}(\eta)\left[\partial_{\xi} \varphi_{m}^{(+)}(\eta, \xi)\right]_{\xi=\xi_{B}}
$$

Thus, calculating the Bogoliubov coefficients amounts to evaluate the wave functions at the brane position, $\chi_{\kappa}\left(\xi_{B}\right)$, and calculate integrals involving Bessel functions.

\subsection{In-out Bogoliubov coefficients: zero mode to zero mode}

We begin with the calculation of the Bogoliubov coefficients in the case where both initial and final wave functions are the zero modes. The final wave function is given by eq. (39), and in coordinates $(\eta, \xi)$ it is

$$
\varphi_{0}^{( \pm)}=-\eta_{0} \sinh \xi_{B} \frac{k^{\frac{3}{2}}}{\sqrt{p}} \mathrm{e}^{ \pm i p \eta \cosh \xi}
$$

whereas the initial wave function is defined in eq. (18).

Collecting all factors, we obtain from the representation (53)

$$
v_{0,0}=C_{1} \eta_{0} \int_{-\infty}^{\eta_{0}} \frac{d \eta}{\eta^{3}}\left(\eta-\frac{i}{p}\right) \mathrm{e}^{-i p \eta\left(1+\cosh \xi_{B}\right)}
$$


For momenta obeying (37) and (38), the integral in (56) is saturated at $\eta \approx \eta_{0}$. We find

$$
v_{0,0}=\frac{i C_{1}}{2 p \eta_{0}}
$$

which means that at small $H / k$, the number of zero-mode gravitons created from fluctuations in the zero mode state is

$$
n_{0,0}=\frac{1}{4 p^{2} \eta_{0}^{2}}, \quad \sinh \xi_{B} \equiv \frac{H}{k} \ll 1
$$

whereas at large $H / k$ one has instead

$$
n_{0,0}=\frac{3}{8 p^{2} \eta_{0}^{2}} \sinh \xi_{B}=\frac{3}{8 p^{2} \eta_{0}^{2}} \frac{H}{k}, \quad \sinh \xi_{B} \equiv \frac{H}{k} \gg 1
$$

In a similar way we get

$$
u_{0,0}=-\frac{i C_{1}}{2 p \eta_{0}}
$$

These expressions coincide with the result of calculation in four dimensional theory (see Appendix 3, eqs. (121) and (122)), up to overall enhancement factor $C_{1}$. This is in accord with Ref. [5]. It is worth noting that the relative phase of $u_{0,0}$ and $v_{0,0}$ coincides too (this relative phase enters the correlators of the gravitational perturbations in the postinflationary epoch).

We note that when calculating the two-point correlators of the gravitational perturbations after inflation, the overall factor of $z_{B}^{2} k^{3}$ will appear due to normalization in (39). This factor may be written as $k\left(H \eta_{0}\right)^{2} \equiv k a^{2}$. The factor $k$ here accounts for the distinction between the five-dimensional field and effective four-dimensional field (i.e., the distinction between the five-dimensional gravitational constant and four-dimensional Newton's constant [1]), whereas the factor $a^{2}$ exists also in four-dimensional theory (see Appendix 3, eq.(117)).

We conclude that the (zero mode)-(zero mode) contribution matches the four-dimensional calculation, up to the factor $C_{1}$. 


\subsection{Zero mode to KK modes}

Let us now calculate the Bogoliubov coefficients from initial zero mode to final KK modes, which describe the creation of gravitons in continuum from zero-point fluctuations in the zero mode of $\mathrm{dS}_{4}$ slicing. Before performing the calculation, we point out that the number of created KK gravitons is given by the integral

$$
\int d m\left|v_{m, 0}\right|^{2}=\int \frac{d m}{m}\left|\sqrt{m} v_{m, 0}\right|^{2}
$$

Hence, the quantity of interest is

$$
\sqrt{m} v_{m, 0}
$$

as it is this quantity that characterizes the number of created particles in a decimal interval of KK masses.

We insert (18) and (41) into expression (53) and obtain

$$
v_{m, 0}=-\frac{i C_{1}}{\sinh ^{2} \xi_{B} \sqrt{2 p \omega}} I_{m, 0}
$$

where

$$
I_{m, 0}=\int_{\left|\eta_{0}\right|}^{\infty} \frac{d \eta}{\eta^{4}} \mathrm{e}^{i p \eta}\left(\eta+\frac{i}{p}\right)\left(\frac{\partial}{\partial \xi}\left[\mathrm{e}^{i \omega \eta \cosh \xi} \varphi_{m}(\eta \sinh \xi)\right]\right)_{\xi=\xi_{B}}
$$

(we changed the integration variable from $\eta$ to $-\eta$ ). For $p$ and $m$ satisfying (37), (38), (43) and (44), we use the expansion of $\varphi_{m}$ at low values of argument, i.e., we insert

$$
\varphi_{m}(\eta \sinh \xi)=-z_{B}^{2} \sqrt{\frac{m}{2}}
$$

and obtain, taking into account that $z_{B}=\left|\eta_{0}\right| \sinh \xi_{B}$, that

$$
I_{m, 0}=\frac{\omega}{2 p} \sqrt{\frac{m}{2}} \sinh ^{3} \xi_{B}
$$

We get finally

$$
\sqrt{m} v_{m, 0}=\frac{-i C_{1}}{4} \frac{m \sqrt{\omega}}{p^{3 / 2}} \sinh \xi_{B}
$$

The spectrum rises towards high momenta (recall that $v_{0,0} \propto p^{-1}$ corresponds to the "flat spectrum"), but the (zero mode)-KK contribution is suppressed, as compared to the (zero 
mode)-(zero mode) result. In terms of the number of created particles, the suppression factor is of order $\left(p\left|\eta_{0}\right| \sinh \xi_{B}\right)^{2}$ or $\left(m\left|\eta_{0}\right| \sinh \xi_{B}\right)^{2}$, which is small in virtue of eqs. (38) or (44). Hence, the creation of KK gravitons from the fluctuations in the zero mode state is a subdominant process for soft gravitons. We note in passing that for gravitons of higher spatial momenta, the situation is different: we show in Appendix 4 that the creation of KK gravitons from the zero mode fluctuations dominates in that case.

\subsection{KK to zero mode}

Let us now consider KK gravitons as initial states and zero mode as the final state. The final zero mode is again given by eq. (55), while initial KK modes are described in subsection 2.2 and Appendix 2.

We are going to calculate the integral (53). Using explicit form of the zero mode (55), we write

$$
v_{0, \kappa}=-\frac{\sqrt{p\left|\eta_{0}\right|}}{\sinh \xi_{B}} \cdot \chi_{\kappa}\left(\xi_{B}\right) \cdot k^{-\frac{3}{2}} \sqrt{\left|\eta_{0}\right|} \int_{\left|\eta_{0}\right|}^{\infty} \frac{d \eta}{\eta^{3}} \psi_{\kappa}^{(-)}(-\eta) \mathrm{e}^{i p \eta \cosh \xi_{B}}
$$

where we again changed the integration variable from $\eta$ to $-\eta$.

Let us begin with the integral over conformal time

$$
I_{0, \kappa}=k^{-\frac{3}{2}} \sqrt{\eta_{0}} \int_{\left|\eta_{0}\right|}^{\infty} \frac{d \eta}{\eta^{3}} \psi_{\kappa}^{(-)}(-\eta) \mathrm{e}^{i p \eta \cosh \xi_{B}}
$$

With the explicit form of the time functions given in subsection 2.2, this integral can be written as follows,

$$
I_{0, \kappa}=\frac{\sqrt{\pi}}{2} \int_{1}^{\infty} \frac{d u}{u^{3 / 2}} \quad \mathrm{e}^{i p\left|\eta_{0}\right| \cosh \xi_{B} \cdot u} \mathrm{e}^{-\frac{\pi \kappa}{2}} H_{i \kappa}^{(1)}\left(p\left|\eta_{0}\right| \cdot u\right)
$$

At small $p\left|\eta_{0}\right|$ and $p\left|\eta_{0}\right| \sinh \xi_{B}$ this integral is again saturated at its lower limit, $u=1$. We make use of the representation

$$
\mathrm{e}^{-\frac{\pi \kappa}{2}} H_{i \kappa}^{(1)}\left(p\left|\eta_{0}\right| \cdot u\right)=\frac{\mathrm{e}^{\frac{\pi \kappa}{2}} J_{i \kappa}\left(p\left|\eta_{0}\right| \cdot u\right)-\mathrm{e}^{-\frac{\pi \kappa}{2}} J_{-i \kappa}\left(p\left|\eta_{0}\right| \cdot u\right)}{\sinh (\pi \kappa)}
$$


and expand in $p \eta_{0}$. We obtain

$$
I_{0, \kappa}=\frac{\sqrt{\pi}}{2} \cdot \frac{\mathrm{e}^{\pi \kappa / 2}}{\sinh (\pi \kappa)} \frac{1}{(1 / 2-i \kappa) \Gamma(1+i \kappa)} \cdot\left(\frac{p\left|\eta_{0}\right|}{2}\right)^{i \kappa}+(\kappa \rightarrow-\kappa)
$$

At small $\kappa$ this expression behaves as

$$
I_{0, \kappa}=\frac{2 i}{\sqrt{\pi}} \cdot \frac{1}{\kappa} \sin \left[\kappa \log \left(\frac{p\left|\eta_{0}\right|}{2}\right)\right], \quad \kappa \ll 1
$$

while at large $\kappa$ it behaves as

$$
I_{0, \kappa}=\frac{\mathrm{e}^{i \frac{\pi}{4}}}{\sqrt{2}} \cdot \frac{1}{\kappa^{3 / 2}} \mathrm{e}^{i \kappa \log \left(p\left|\eta_{0}\right| / 2\right)+i \kappa(1-\log \kappa)}, \quad \kappa \gg 1 .
$$

It remains to use the formulas for spatial functions $\chi_{\kappa}\left(\xi_{B}\right)$ to obtain the Bogoliubov coefficients. Let us begin with the case $\sinh \xi_{B} \equiv H / k \ll 1$. The contributions to the number of created zero-mode gravitons, coming from zero-point fluctuations with small and large $\kappa$, have different form. At $\kappa \ll \sinh ^{-1} \xi_{B}$ we use eq. (107) and obtain

$$
v_{0, \kappa}=-\frac{1}{\sqrt{2}}\left|\frac{\frac{1}{4}+i \frac{\kappa}{2}}{\frac{3}{4}+i \frac{\kappa}{2}}\right| \cdot \sqrt{p\left|\eta_{0}\right|} \sinh \xi_{B} \sqrt{\kappa \tanh \kappa} \cdot I_{0, \kappa}, \quad \kappa \ll \sinh ^{-1} \xi_{B}
$$

At $\kappa \gg \sinh ^{-1} \xi_{B}$, equation (113) with $\cos \theta_{\kappa}=1$ is appropriate, and we find

$$
v_{0, \kappa}=-\frac{1}{\sqrt{\pi}} \sqrt{p\left|\eta_{0}\right| \sinh \xi_{B}} \cdot I_{0, \kappa}
$$

Making use of eqs. (73) and (74) we find that the integral for the number of created gravitons is saturated at $\kappa \ll \sinh ^{-1} \xi_{B}$ and is of order

$$
\int d \kappa\left|v_{0, \kappa}\right|^{2} \sim p\left|\eta_{0}\right| \sinh ^{2} \xi_{B}, \quad \sinh \xi_{B} \ll 1
$$

Clearly, it is suppressed as compared to the (zero mode)-(zero mode) contribution to the number of created gravitons, eq. (58), by a factor $\left(p\left|\eta_{0}\right|\right)^{3} \sinh ^{2} \xi_{B}=\left(p_{\text {phys }} / k\right)^{2} \cdot\left(p_{\text {phys }} / H\right)$.

Let us now turn to the case $\sinh \xi_{B} \equiv H / k \gg 1$. In this case we use eq. (113) and find

$$
v_{0, \kappa} \sim \sqrt{p\left|\eta_{0}\right| \sinh \xi_{B}} \cdot I_{0 \kappa} \cos \theta_{\kappa}
$$


For $\kappa \ll 1$ and $\kappa \gg 1$, one has $\cos \theta_{\kappa}=2 \kappa / 3$ and $\cos \theta_{\kappa}=1$, respectively. Making use of eqs. (73) and (74) we find that the integral for the number of created gravitons is of order

$$
\int d \kappa\left|v_{0, \kappa}\right|^{2} \sim p\left|\eta_{0}\right| \sinh \xi_{B}, \quad \sinh \xi_{B} \gg 1
$$

Again, it is suppressed as compared to the (zero mode)-(zero mode) contribution, eq. (59), but now by a factor $\left(p\left|\eta_{0}\right|\right)^{3}=\left(p_{\text {phys }} / H\right)^{3}$.

We conclude that the contribution of the zero-point fluctuations of the KK modes into the production of soft gravitons in the zero mode state is negligible.

\subsection{Continuum to continuum}

Finally, we calculate the Bogoliubov coefficients in the case where both initial and final wave functions belong to coninuous spectra. We are again interested in the quantity $\sqrt{m} v_{m, \kappa}$ (see the discussion in the beginning of subsection 3.4).

The expression (53) now reads

$$
v_{m, \kappa}=\frac{1}{\sqrt{2 \omega}} \frac{\chi_{\kappa}\left(\xi_{B}\right)}{\sinh ^{3} \xi_{B}} \int_{\left|\eta_{0}\right|}^{\infty} \frac{d \eta}{\eta^{5 / 2}} \mathrm{e}^{-\pi \kappa / 2} H_{i \kappa}^{(1)}(p \eta) \frac{\partial}{\partial \xi}\left[\mathrm{e}^{i \omega \eta \cosh \xi} \varphi_{m}(\eta \sinh \xi)\right]_{\xi=\xi_{B}}
$$

We again use the expression (65) and obtain

$$
\sqrt{m} v_{m, \kappa}=-\frac{i}{2} \cdot\left|\eta_{0}\right|^{3 / 2} \chi_{\kappa}\left(\xi_{B}\right) m \sqrt{\omega} \int_{1}^{\infty} \frac{d u}{u^{3 / 2}} \mathrm{e}^{i \omega\left|\eta_{0}\right| u \cosh \xi_{B}} \mathrm{e}^{-\pi \kappa / 2} H_{i \kappa}^{(1)}\left(p\left|\eta_{0}\right| \cdot u\right)
$$

The integral here is the same as in eq. (70), and it is dominated by the lower limit of the integration. Comparing eq. (81) with eqs. (68) and (70) we find that at small $p$ and $m$, the creation of KK gravitons from KK zero-point fluctuations is further suppressed as compared to the case of subsection 3.5. For the number of created gravitons, the suppression factor is of order $\left(p\left|\eta_{0}\right| \sinh y_{B}\right)^{2}$ or $\left(m\left|\eta_{0}\right| \sinh y_{B}\right)^{2}$. Thus, the "KK to KK" contribution to the creation of soft gravitons is completely negligible. 


\subsection{Discussion}

We have found that the main source of created gravitons is the initial zero-point fluctuations in the zero-mode state existing in the frame with $\mathrm{dS}_{4}$ slicing. The contributions of initial KK modes are negligible in all cases. As shown in Appendix 4, this general property holds not only for soft gravitons considered in this section, but also for gravitons of somewhat higher spatial momenta.

Soft gravitons obeying (37) and (38) (and also (43) and (44) for KK modes) are created predominantly in the final zero-mode state. This is not, however, the general property of this system: we find in Appendix 4 that the situation is inverse for gravitons whose wavelengths at the end of inflation are shorter than the $\operatorname{adS}_{5}$ radius. For the moment, the novel feature of the inflationary brane-world model - the creation of KK gravitons, or, in other words, the emission of five-dimensional gravitons into the bulk - appears of academic interest only.

The authors are indebted to N. Arkani-Hamed, P. Binetryi, N. Deruelle, S. Dubovsky, D. Langlois and P. Tinyakov for helpful discussions. This work has been supported in part by RFBR grant 99-02-18410, CPG and SSLSS grant 00-15-96626, CRDF grant (award RP12103), Swiss Science Foundation grant 7SUPJ062239. The work of D.G. was supported in part also under RFBR grant 01-02-06035. The work of S.S. was supported in part also under RFBR grant 01-02-06034.

\section{Appendix 1. Smallness of graviton momenta.}

We consider in the main text the creation of gravitons whose physical momentum at the end of inflation is smaller than the inverse $\mathrm{adS}_{5}$ radius,

$$
p_{\text {phys }} \ll k
$$

Here we argue that only these gravitons are of interest from the point of view of CMB anisotropy. For this purpose, we estimate the minimum possible present momentum $p_{0}$ of 
gravitons which at the end of inflation have

$$
p_{\text {phys }}=k
$$

We will see that in any feasible scenario, the present wavelength $1 / p_{0}$ is too short to be relevant for $\mathrm{CMB}$ anisotropy.

In the first place, we are dealing with gravitons which are superhorizon by the end of inflation,

$$
p_{\text {phys }} \ll H
$$

Thus, we only have to consider the case

$$
H \gg k
$$

There is nothing wrong with this case, but the cosmological evolution is somewhat different from the conventional four-dimensional cosmology. As shown in Ref. [10], instead of the conventional Friedmann equation one has in this case

$$
H^{2}=\frac{16 \pi^{2}}{9} \cdot \kappa_{5}^{4} \rho^{2}
$$

where $\kappa_{5}^{2}=M_{(5)}^{-3}$ is the five-dimensional gravitational constant. With $M_{(5)}^{3}=k M_{P l}^{2}$ this means

$$
\rho_{e . i .} \sim H k M_{P l}^{2}
$$

This determines the energy density just after inflation (hence the subscript, meaning "end of inflation").

Let us set an upper limit on the redshift from the end of inflation to today. Consider an extreme case of very long post-inflationary preheating, at which the equation of state corresponds to pressureless matter. Let us assume, again as an extreme case, that this regime terminates only at the nucleosynthesis epoch, $T_{N S} \sim 10 \mathrm{MeV}$. Then

$$
z_{e . i .} \equiv \frac{a_{0}}{a_{e . i .}}=\frac{a_{0}}{a_{N S}} \frac{a_{N S}}{a_{e . i .}} \sim \frac{T_{N S}}{T_{0}}\left(\frac{\rho_{e . i .}}{T_{N S}^{4}}\right)^{1 / 3}
$$


where the subscript 0 refers to today.

Now, the Hubble parameter at inflation, $H$, must be smaller than the fundamental scale $M_{(5)}$, i.e.,

$$
H<\left(k M_{P l}^{2}\right)^{1 / 3}
$$

In fact, a stronger bound comes from gravity waves themselves, as their amplitude (coming from (zero mode) - (zero mode) Bogoliubov coefficients, with account of $C_{1} \sim \sqrt{H / k}$ ) is

$$
\delta \sim \frac{H}{M_{P l}} \sqrt{\frac{H}{k}}
$$

It must be smaller than about $10^{-3}$, so instead of (86) we have

$$
H<\left(\delta^{2} k M_{P l}^{2}\right)^{1 / 3}
$$

where $\delta \sim 10^{-3}$.

For the present wavelength of a mode whose wave number just after inflation was equal to $k$ we have

$$
l_{0} \equiv \frac{1}{p_{0}}=\frac{z_{e . i .}}{k}=\frac{1}{k} \frac{T_{N S}}{T_{0}}\left(\frac{k H M_{P l}^{2}}{T_{N S}^{4}}\right)^{1 / 3}
$$

The smaller $k$, the larger $l_{0}$, even if one takes into account (88). For $k^{-1} \sim 1 \mathrm{~mm}$ one obtains from (88)

$$
H<3 \cdot 10^{6} \mathrm{GeV}
$$

and then from (89) one finds

$$
l_{0}<10^{23} \mathrm{~cm} \sim 30 \mathrm{kpc}
$$

As claimed, this is too short scale to be relevant for the CMB anisotropy.

In all above estimates we pushed everything to extreme, in more realistic cases the scale $l_{0}$ should be much smaller.

\section{Appendix 2. Graviton modes in the frame with de Sitter slicing}




\section{Small $\sinh \xi_{B}$ and $\kappa \sinh \xi_{B}$}

In terms of the variable $u=\cosh ^{2} \xi$, eq.(23) is the hypergeometric equation

$$
u(1-u) \partial_{u}^{2} \chi+\left(\frac{1}{2}+\frac{1}{2} u\right) \partial_{u} \chi-\left(\frac{9}{16}+\frac{\kappa^{2}}{4}\right) \chi=0
$$

(the subscript in $\chi_{\kappa}$ is omitted). Another way to cast eq.23) into hypergeometric form is to introduce the variable $v=-\sinh ^{2} \xi$. Then one has

$$
v(1-v) \partial_{v}^{2} \chi+\left(-1+\frac{1}{2} v\right) \partial_{v} \chi-\left(\frac{9}{16}+\frac{\kappa^{2}}{4}\right) \chi=0
$$

It is then convenient to choose two linear independent solutions as follows. The first solution is

$$
\chi_{1}=F\left(\alpha, \beta ; \frac{1}{2} ; \cosh ^{2} \xi\right)
$$

where

$$
\alpha=-\frac{3}{4}+i \frac{\kappa}{2}, \quad \beta=-\frac{3}{4}-i \frac{\kappa}{2}
$$

and the hypergeometric function $F$ should be evaluated at a fixed side of the branch cutf extending from 1 to $\infty$ (say, above the real axis). This form of the solution is clear from the representation (92). The form of the second solution comes from the representation (93)

$$
\chi_{2}=\sinh ^{4} \xi F\left(\frac{1}{2}-\alpha, \frac{1}{2}-\beta ; 3 ;-\sinh ^{2} \xi\right)
$$

The reason to choose this particular pair of solutions is that they behave quite differently at small $\xi$ : the first one behaves as

$$
\chi_{1}=\frac{\Gamma(1 / 2)}{\Gamma(1 / 2-\alpha) \Gamma(1 / 2-\beta)}\left(1+\alpha \beta \sinh ^{2} \xi+O\left(\sinh ^{4} \xi \log (\sinh \xi)\right)\right)
$$

whereas the second one behaves as

$$
\chi_{2}=\sinh ^{4} \xi
$$

As a by-product, these formulae tell that $\chi_{1}$ and $\chi_{2}$ are indeed linear independent.

\footnotetext{
${ }^{4}$ Another choice of the side of the branch cut would correspond to a linear combination of the original solution and the second one, $\chi_{2}$.
} 
We now write the required solution in the form

$$
\chi=A_{1} \chi_{1}+A_{2} \chi_{2}
$$

and impose the Neumann condition (17). We first perform the calculations assuming that

$$
\sinh \xi_{B} \ll 1 \text { and } \kappa \sinh \xi_{B} \ll 1
$$

The opposite case is easy and will be considered later. In the case (100) we use the small- $\xi$ expansions (97) and (98) near $\xi=\xi_{B}$ and obtain

$$
A_{1}=-A_{2} \cdot \sinh ^{2} \xi_{B} \cdot \frac{2 \Gamma(1 / 2-\alpha) \Gamma(1 / 2-\beta)}{\alpha \beta \Gamma(1 / 2)}
$$

Note that $A_{1}$ is small compared to $A_{2}$ for small $\sinh \xi_{B}$.

We now wish to calculate the normalization factor. The following normalization of continuum modes is appropriate,

$$
\int \frac{d \xi}{\sinh ^{3} \xi} \chi_{\kappa}^{*} \chi_{\kappa^{\prime}}=\delta\left(\kappa-\kappa^{\prime}\right)
$$

As is usual for continuum spectrum, the normalization factor is determined by large- $\xi$ asymptotics of the solution. It is straightforward to find the asymptotics of the first solution,

$$
\begin{aligned}
\chi_{1}= & \frac{\Gamma(1 / 2) \Gamma(-i \kappa)}{\Gamma(-3 / 4-i \kappa / 2) \Gamma(5 / 4-i \kappa / 2)}(\cosh \xi)^{\frac{3}{2}-i \kappa} \mathrm{e}^{i \theta} \\
+ & \frac{\Gamma(1 / 2) \Gamma(i \kappa)}{\Gamma(-3 / 4+i \kappa / 2) \Gamma(5 / 4+i \kappa / 2)}(\cosh \xi)^{\frac{3}{2}+i \kappa} \mathrm{e}^{i \theta^{\prime}}
\end{aligned}
$$

where phases $\theta$ and $\theta^{\prime}$ are not important for our purposes. The asymptotics of the second solution is

$$
\begin{aligned}
\chi_{2}= & \frac{\Gamma(3) \Gamma(-i \kappa)}{\Gamma(5 / 4-i \kappa / 2) \Gamma(7 / 4-i \kappa / 2)}(\sinh \xi)^{\frac{3}{2}-i \kappa} \\
+ & \frac{\Gamma(3) \Gamma(i \kappa)}{\Gamma(5 / 4+i \kappa / 2) \Gamma(7 / 4+i \kappa / 2)}(\sinh \xi)^{\frac{3}{2}+i \kappa}
\end{aligned}
$$

At large $\xi$ one neglects $A_{1} \chi_{1}$ term in (99). Making use of (103), one finds

$$
A_{2}=-\frac{1}{2 \sqrt{\pi}}\left|\frac{\Gamma(5 / 4+i \kappa / 2) \Gamma(7 / 4+i \kappa / 2)}{\Gamma(3) \Gamma(i \kappa)}\right|
$$


(sign here is chosen for future convenience). This expression can be simplified by making use of the identities,

$$
\begin{aligned}
\left|\Gamma\left(\frac{5}{4}+i \frac{\kappa}{2}\right) \Gamma\left(\frac{7}{4}+i \frac{\kappa}{2}\right)\right|^{2} & =\left|\left(\frac{1}{4}+i \frac{\kappa}{2}\right) \Gamma\left(1-\frac{3}{4}+i \frac{\kappa}{2}\right) \Gamma\left(1+\frac{3}{4}+i \frac{\kappa}{2}\right)\right|^{2} \\
& =\frac{2 \pi^{2}}{\cosh (\pi \kappa)}\left|\frac{1}{4}+i \frac{\kappa}{2}\right|^{2}\left|\frac{3}{4}+i \frac{\kappa}{2}\right|^{2} \\
& |\Gamma(i \kappa)|^{2}=\frac{\pi}{\kappa \sinh (\pi \kappa)}
\end{aligned}
$$

We find

$$
A_{2}=-\frac{1}{2} \sqrt{\frac{\kappa}{2} \tanh \kappa}\left|\frac{1}{4}+i \frac{\kappa}{2}\right|\left|\frac{3}{4}+i \frac{\kappa}{2}\right|
$$

For the calculation of the Bogoliubov coefficients we need the value of the wave function at the brane position, $\xi=\xi_{B}$. Since $A_{2} \chi_{2}\left(\xi_{B}\right) \sim A_{2} \sinh ^{4} \xi_{B}$ and $A_{1} \chi_{1}\left(\xi_{B}\right) \sim A_{2} \kappa^{-2} \sinh ^{2} \xi_{B}$, the part $A_{1} \chi_{1}$ is dominant on the brane. Making use of eqs.(97) and (101) we find

$$
\begin{aligned}
\chi_{\kappa}\left(\xi_{B}\right) & =A_{1} \chi_{1}\left(\xi_{B}\right) \\
& =-A_{2} \frac{2}{\left|\frac{3}{4}+i \frac{\kappa}{2}\right|^{2}} \cdot \sinh ^{2} \xi_{B} \\
& =\frac{1}{\sqrt{2}} \sqrt{\kappa \tanh \kappa} \frac{\frac{1}{4}+i \frac{\kappa}{2} \mid}{\left|\frac{3}{4}+i \frac{\kappa}{2}\right|} \cdot \sinh ^{2} \xi_{B}
\end{aligned}
$$

We recall that these formulae are valid provided the relations (100) are satisfied. We now turn to the opposite case.

\section{Large $\sinh \xi_{B}$ and/or $\kappa \sinh \xi_{B}$}

In the opposite case,

$$
\sinh \xi_{B} \gg 1 \text { and/or } \kappa \sinh \xi_{B} \gg 1
$$

it is convenient to introduce the wave function

$$
\tilde{\chi}(\xi)=\chi(\xi) \sinh ^{-3 / 2} \xi
$$

and cast equation (23) in the form of the Schrödinger equation

$$
\left(-\partial_{\xi}^{2}+\frac{15}{4 \sinh ^{2} \xi}\right) \tilde{\chi}=\kappa^{2} \tilde{\chi}
$$


In the case of interest, the potential term in eq.(110) may be neglected, and the solution (normalized to delta-function) becomes

$$
\chi=\frac{1}{\sqrt{\pi}} \sinh ^{3 / 2} \xi \cos \left[\kappa\left(\xi-\xi_{B}\right)+\theta_{\kappa}\right]
$$

The phase here is determined from the Neumann boundary condition on the brane, eq.(17). One finds

$$
\tan \theta_{\kappa}=\frac{3 \cosh \xi_{B}}{2 \kappa \sinh \xi_{B}}
$$

Thus, the value of the wave function at the brane position is

$$
\chi_{\kappa}\left(\xi_{B}\right)=\frac{1}{\sqrt{\pi}} \sinh ^{3 / 2} \xi_{B} \cos \theta_{\kappa}
$$

Note that for parameters obeying eq. (108), the value of the wave function on the brane is always smaller than $\frac{1}{\sqrt{\pi}} \sinh ^{3 / 2} \xi_{B}$.

\section{Appendix 3. Bogoliubov coefficients in four-dimen- sional theory.}

In the four-dimensional theory one considers the metric which is de Sitter at inflationary stage

$$
d s^{2}=\frac{1}{(H \eta)^{2}}\left(d \eta^{2}-d \mathbf{x}^{2}\right), \quad \eta<\eta_{0}
$$

and flat afterwards,

$$
d s^{2}=\frac{1}{\left(H \eta_{0}\right)^{2}}\left(d \eta^{2}-d \mathbf{x}^{2}\right), \quad \eta>\eta_{0}
$$

Properly normalized solutions in the de Sitter and flat regions are

$$
\phi^{( \pm)}=\frac{H}{\sqrt{2 p}} \mathrm{e}^{ \pm i p \eta}\left(\eta \pm \frac{i}{p}\right)
$$

and

$$
\varphi^{(-)}=\frac{H}{\sqrt{2 p}} \eta_{0} \mathrm{e}^{-i p \eta}
$$

respectively. 
In the de Sitter region, one writes the solution that becomes $\varphi^{(-)}$at late times as a linear combination

$$
\Phi=u^{*} \phi^{(-)}+v \phi^{(+)}
$$

and finds the Bogoliubov coefficients by matching this solution and its derivative to $\varphi^{(-)}$at $\eta=\eta_{0}$. In this way one obtains

$$
\begin{gathered}
v=\mathrm{e}^{-2 i p \eta_{0}} \frac{i}{2 p \eta_{0}} \\
u=1-\frac{i}{2 p \eta_{0}}
\end{gathered}
$$

At small $p \eta_{0}$ (physical momentum just after inflation small comared to $H$ ), these become

$$
\begin{gathered}
v=\frac{i}{2 p \eta_{0}} \\
u=-\frac{i}{2 p \eta_{0}}
\end{gathered}
$$

This is the standard "flat spectrum".

\section{Appendix 4. Creation of gravitons of higher mo- menta}

At $H \gg k$, i.e. $\sinh \xi_{B} \gg 1$, there exists a range of momenta in which the gravitons are superhorizon at the end of inflation,

$$
p\left|\eta_{0}\right| \ll 1
$$

and yet their physical momentum at that time exceeds the inverse $\operatorname{adS}_{5}$ radius,

$$
p\left|\eta_{0}\right| \sinh \xi_{B} \gg 1
$$

Here we present for completeness the results of calculations of the Bogoliubov coefficients in this case. For the masses of final KK gravitons we assume also

$$
m\left|\eta_{0}\right| \ll 1, \quad m\left|\eta_{0}\right| \sinh \xi_{B} \gg 1
$$

so that their physical frequency is small compared to $H$ and large compared to $k$ at the end of inflation. 


\section{Zero mode to zero mode}

The integral (56) is equal to

$$
v_{0,0}=\frac{C_{1}}{p \eta_{0}} \frac{\mathrm{e}^{i \eta_{0} p \cosh \xi_{B}}}{p \eta_{0} \cosh \xi_{B}}
$$

where, as before, $C_{1}=\sqrt{3 \sinh \xi_{B} / 2}$ at large $\sinh \xi_{B}$. Comparing this result with eq. (57) and recalling (123), we see that the spectrum of created gravitons is damped at higher momenta due to the extra factor $\left(p \eta_{0} \cosh \xi_{B}\right)^{-2}$ appearing in $n_{0,0} \equiv\left|v_{0,0}\right|^{2}$.

\section{Zero mode to KK modes}

To evaluate the integral (64), we use the approximation

$$
\begin{array}{r}
\left.\frac{\partial}{\partial \xi}\left[\mathrm{e}^{i \omega \eta \cosh \xi} \varphi_{m}(\eta \sinh \xi)\right]\right|_{\xi=\xi_{B}}=\left(\eta \sinh \xi_{B}\right)^{5 / 2} \frac{\mathrm{e}^{i \omega \eta \cosh \xi_{B}}}{\sqrt{\pi}} \\
\times\left(i \omega \cos \left(m\left(\left|\eta_{0}\right|-\eta\right) \sinh \xi_{B}\right)+m \operatorname{coth} \xi_{B} \sin \left(m\left(\left|\eta_{0}\right|-\eta\right) \sinh \xi_{B}\right)\right)
\end{array}
$$

We obtain

$$
\sqrt{m} v_{m, 0}=-\frac{\sqrt{3}}{2 \sqrt{2 \pi}} \operatorname{coth} \xi_{B} \mathrm{e}^{i\left|\eta_{0}\right| \omega \cosh \xi_{B}} \frac{\sqrt{m \eta_{0}}}{\eta_{0}^{2}\left(\omega^{2} \operatorname{coth}^{2} \xi_{B}-m^{2}\right)} \sqrt{\frac{p}{\omega}}
$$

This result is to be compared with eq. (67). We see that the spectrum no longer increases towards high momenta; instead, it turns down at large $p$ and $m$. It is of interest also to compare eqs. (127) and (125) and find that for higher momentum gravitons

$$
\frac{\left|v_{0,0}\right|^{2}}{\left|\sqrt{m} v_{m, 0}\right|^{2}} \sim \frac{1}{p\left|\eta_{0}\right| \cosh \xi_{B}}
$$

This means that as far as the zero-mode initial fluctuations are concerned, the production of KK gravitons dominates over the production of the zero-mode gravitons in the range of momenta considered in this Appendix. This is in contrast to the case studied in the main text.

\section{KK to zero mode}

The Bogoliubov coefficient reads

$$
v_{0, \kappa}=-\frac{\sqrt{p\left|\eta_{0}\right|}}{\sinh \xi_{B}} \cdot \chi_{\kappa}\left(\xi_{B}\right) \cdot I_{0, \kappa}
$$


where $\chi_{\kappa}\left(\xi_{B}\right)$ is given by Eq. (113) and

$$
I_{0, \kappa}=\frac{\sqrt{\pi}}{2} \int_{1}^{\infty} \frac{d u}{u^{3 / 2}} \quad \mathrm{e}^{i p\left|\eta_{0}\right| \cosh \xi_{B} \cdot u} \mathrm{e}^{-\frac{\pi \kappa}{2}} H_{i \kappa}^{(1)}\left(p\left|\eta_{0}\right| \cdot u\right)
$$

This integral is again saturated at the lower limit of integration, so in the integrand we substitute the expression for the Hankel function at small value of its argument. In this way we obtain

$$
I_{0, \kappa}=i \frac{\sqrt{\pi}}{2} \frac{\mathrm{e}^{i p\left|\eta_{0}\right| \cosh \xi_{B}}}{\sinh (\pi \kappa)} \frac{1}{p\left|\eta_{0}\right| \cosh \xi_{B}}\left(\left(\frac{p\left|\eta_{0}\right|}{2}\right)^{i \kappa} \frac{\mathrm{e}^{\frac{\pi \kappa}{2}}}{\Gamma(1+i \kappa)}-(\kappa \rightarrow-\kappa)\right)
$$

The final estimate for the number of created gravitons is

$$
\int d \kappa\left|v_{0, \kappa}\right|^{2} \sim\left(p\left|\eta_{0}\right| \sinh \xi_{B}\right)^{-1}
$$

Comparing this estimate with eq. (79) we find that the spectrum is again damped at higher momenta: the ratio of eqs. (128) and (79) is of order $\left(p\left|\eta_{0}\right| \sinh \xi_{B}\right)^{-2}$. Also, it follows from eqs. (125) and (128) that the production of the zero mode gravitons from KK modes is suppressed by the factor $\left(p\left|\eta_{0}\right|\right)^{3}$ in comparison with the production of zero mode gravitons from the fluctuations in the zero mode. This suppression is the same as for soft gravitons considered in subsection 3.5.

\section{Continuum to continuum}

The Bogoliubov coefficients are given by eq. (80) where $\chi_{\kappa}\left(\xi_{B}\right)$ is determined by eq. (113), and the approximation (126) can be used. Thus, one has to calculate the integral

$$
\begin{gathered}
v_{m, \kappa}=\frac{\left|\eta_{0}\right|}{\pi} \sinh \xi_{B} \cos \theta_{\kappa} \frac{e^{-\pi \kappa / 2}}{\sqrt{\omega}} \int_{1}^{\infty} d u H_{i \kappa}^{(1)}\left(p\left|\eta_{0}\right| \cdot u\right) \mathrm{e}^{i \omega\left|\eta_{0}\right| \cosh \xi_{B} \cdot u} \\
\times\left(i \omega \cos \left(m\left|\eta_{0}\right|(1-u) \sinh \xi_{B}\right)+m \operatorname{coth} \xi_{B} \sin \left(m\left|\eta_{0}\right|(1-u) \sinh \xi_{B}\right)\right)
\end{gathered}
$$

It is saturated again at the lower limit of integration. We find

$$
\begin{aligned}
v_{m, \kappa} & =-\frac{1}{2} \frac{\cos \theta_{\kappa}}{\sqrt{2 \omega}} \frac{\mathrm{e}^{i \omega\left|\eta_{0}\right| \cosh \xi_{B}}}{\sinh \pi \kappa} \\
& \times\left\{\frac { \omega - m \operatorname { c o t h } \xi _ { B } } { \omega \operatorname { c o t h } \xi _ { B } - m } \left[\frac{\mathrm{e}^{\pi \kappa / 2}}{\Gamma(1+i \kappa)}\left(\frac{p\left|\eta_{0}\right|}{2}\right)^{i \kappa}\left(1-\frac{\kappa}{\left|\eta_{0}\right|\left(\omega \cosh \xi_{B}-m \sinh \xi_{B}\right)}\right)\right.\right. \\
& -(\kappa \rightarrow-\kappa)]+(m \rightarrow-m)\}
\end{aligned}
$$


One can show that this expression leads to the number of created gravitons suppressed by a factor $\left(p\left|\eta_{0}\right| \sinh \xi_{B}\right)^{-3}$ as compared to the case of soft gravitons, see eq. (81). Furthermore, comparing eqs. (129) and (127), one finds that the production of KK modes of higher spatial momenta from KK modes is suppressed by a factor $\left(p\left|\eta_{0}\right|\right)^{3}$ in comparison with the production of the KK modes from the zero mode.

\section{Discussion}

For momenta obeying (123), the dominant effect is the creation of KK gravitons from initial fluctuations in the zero mode. In five-dimensional language, this corresponds to the emission of the five-dimensional gravitons into the bulk. Let us estimate the energy per unit three-volume, emitted into the bulk in the form of superhorizon gravitons,

$$
\epsilon_{\text {superhorizon }}=\frac{1}{a^{4}\left(z_{B}\right)} \int \frac{d m}{m} \frac{d^{3} p}{(2 \pi)^{3}} \omega\left|\sqrt{m} v_{m, 0}\right|^{2}
$$

where the factor $a^{-4}\left(z_{B}\right)$ accounts for the fact that $p$ and $\omega$ are conformal, rather than physical, three-momentum and frequency. Making use of eq. (127), and setting the upper limit of integration equal to $p_{\max } \sim m_{\max } \sim\left|\eta_{0}\right|^{-1}$, we obtain an estimate

$$
\epsilon_{\text {superhorizon }} \sim \frac{1}{a^{4}\left(z_{B}\right) \eta_{0}^{4}} \sim H^{4}
$$

In view of eqs. (84) and (88), we find

$$
\frac{\epsilon_{\text {superhorizon }}}{\rho_{\text {e.i. }}}<\delta^{2} \ll 1
$$

This means that only a small fraction of energy leaks from the brane into the bulk in the form of gravitons with momenta smaller than $H$. The emission into the bulk is a small effect, at least for superhorizon modes.

\section{References}

[1] L. Randall and R. Sundrum, Phys. Rev. Lett. 83 (1999) 4690 hep-th/9906064. 
[2] V. A. Rubakov, hep-ph/0104152.

[3] R. Maartens, D. Wands, B. A. Bassett and I. Heard, Phys. Rev. D 62 (2000) 041301 hep-ph/9912464.

S. Mukohyama, Phys. Rev. D 62 (2000) 084015 hep-th/0004067;

H. Kodama, A. Ishibashi and O. Seto, Phys. Rev. D 62 (2000) 064022 hep-th/0004160;

D. Langlois, Phys. Rev. D 62 (2000) 126012 hep-th/0005025;

C. van de Bruck, M. Dorca, R. H. Brandenberger and A. Lukas, Phys. Rev. D 62 (2000) 123515 hep-th/0005032;

K. Koyama and J. Soda, Phys. Rev. D 62 (2000) 123502 hep-th/0005239];

S. Mukohyama, Class. Quant. Grav. 17 (2000) 4777 hep-th/0006146];

C. Gordon, D. Wands, B. A. Bassett and R. Maartens, Phys. Rev. D 63 (2001) 023506 astro-ph/0009131.

D. Langlois, R. Maartens, M. Sasaki and D. Wands, Phys. Rev. D 63 (2001) 084009 hep-th/0012044.

O. Seto and H. Kodama, Phys. Rev. D 63 (2001) 123506 [hep-th/0012102];

C. van de Bruck and M. Dorca, hep-th/0012073.

K. Koyama and J. Soda, hep-th/0108003.

[4] S. W. Hawking, T. Hertog and H. S. Reall, Phys. Rev. D 62 (2000) 043501 hepth/0003052]; Phys. Rev. D 63 (2001) 083504 [hep-th/0010232].

[5] D. Langlois, R. Maartens and D. Wands, Phys. Lett. B 489 (2000) 259 [hepth/0006007].

[6] P. Kraus, JHEP 9912 (1999) 011 hep-th/9910149];

D. Ida, JHEP 0009 (2000) 014 gr-qc/9912002; 
M. Cvetic and J. Wang, Phys. Rev. D 61, 124020 (2000) [hep-th/9912187;;

S. Mukohyama, T. Shiromizu and K. Maeda, Phys. Rev. D 62 (2000) 024028 [Erratumibid. D 63 (2000) 029901] hep-th/9912287;

P. Bowcock, C. Charmousis and R. Gregory, Class. Quant. Grav. 17 (2000) 4745 [hepth/0007177.

[7] S. Alexander, Y. Ling and L. Smolin, "A thermal instability for positive brane cosmological constant in the Randall-Sundrum cosmologies," hep-th/0106097.

[8] J. Garriga and M. Sasaki, Phys. Rev. D 62 (2000) 043523 [hep-th/9912118].

[9] A. Karch and L. Randall, JHEP 0105 (2001) 008 hep-th/0011156.

[10] P. Binetruy, C. Deffayet, U. Ellwanger and D. Langlois, Phys. Lett. B 477 (2000) 285 [hep-th/9910219]. 